\title{
The story of Landen, the hyperbola and the ellipse
}

Victor H. Moll, Judith L. Nowalsky, Leonardo Solanilla

Victor H. Moll was born in Santiago, Chile. He studied under H. McKean at the Courant Institute and joined the Department of Mathematics at Tulane University in New Orleans. His current mathematical interests lie in the evaluation of definite integrals.

Judith L. Nowalsky was born in New Orleans, Louisiana. She obtained a M.S. in teaching at Loyola University and a M.S. in mathematics at Tulane University in 1998. She is currently an instructor of mathematics at the University of New Orleans.

Leonardo Solanilla was born in Ibague, Tolima, Colombian Andes. He obtained a B.S. from the Universidad de los Andes in Bogota in electrical engineering and a $\mathrm{Ph}$.D. from Tulane University in 1999 . He is presently working at the Universidad del Tolima, Ibague, Colombia.

\section{Introduction}

The problem of rectification of conics was a central question of analysis in the 18th century. The goal of this note is to describe Landen's work on rectifying the arc of a hyperbola in terms of an ellipse and a circle. Naturally, Landen's language is that of his time, in terms of fluents and fluxions, and his arguments are not rigorous in the modern sense.

The main result presented here is a special relation between the length of an ellipse, the length of a hyperbolic segment, and the length of a circle. The proof is based on a generalization of Euler's formula for the lemniscatic curve as described in [4].

Der nachfolgende Beitrag knüpft an einen Artikel der drei selben Autoren gemeinsam mit P.A. Neill über eine gewisse Eigenschaft von Eulers elastischer Kurve (Elem. Math. 55 (2000), 156-162) an. In der nun vorliegenden Arbeit geht es um die Bestimmung der Länge von Hyperbelbögen. Zur Lösung der Aufgabe wird eine Idee von J. Landen aus dem 18. Jahrhundert herangezogen. Damit lässt sich die gesuchte Hyperbelbogenlänge schliesslich mit Hilfe einer Ellipsenbogenlänge ausdrücken, welche in der vorhergehenden Arbeit untersucht wurde. 


\section{The hyperbola}

The arc length of the equilateral hyperbola

$$
h(t)=\sqrt{t^{2}-1}, \quad t \geq 1
$$

starting at $t=1$ is given by

$$
L_{h}(x)=\int_{1}^{x} \sqrt{\frac{2 t^{2}-1}{t^{2}-1}} \mathrm{~d} t
$$

as a function of the terminal point $t=x$. The tangent line to the hyperbola at $t=x$ is

$$
T_{h}(t)=\sqrt{x^{2}-1}+\frac{x}{\sqrt{x^{2}-1}}(t-x),
$$

whose intersection with the $t$-axis is $t=1 / x \in(0,1)$. The line

$$
N_{h}(t)=-\frac{\sqrt{x^{2}-1}}{x} t
$$

is the perpendicular to $T_{h}$ passing through the origin. The lines $T_{h}$ and $N_{h}$ intersect at the point

$$
P_{h}=\left(\frac{x}{2 x^{2}-1},-\frac{\sqrt{x^{2}-1}}{2 x^{2}-1}\right) .
$$

The distance from $(x, h(x))$ to the common point $P_{h}$ is

$$
g_{h}(x)=2 x \sqrt{\frac{x^{2}-1}{2 x^{2}-1}} .
$$

It was observed by Maclaurin, D'Alembert, and Landen that

$$
f_{h}(x):=g_{h}(x)-L_{h}(x)=2 x \sqrt{\frac{x^{2}-1}{2 x^{2}-1}}-\int_{1}^{x} \sqrt{\frac{2 t^{2}-1}{t^{2}-1}} \mathrm{~d} t
$$

is easier to analyze than the arc length $L_{h}(x)$.

Proposition 2.1 Let

$$
F_{h}(z)=\frac{1}{2} \int_{z}^{1} \sqrt{\frac{t}{1-t^{2}}} \mathrm{~d} t
$$

Then

$$
F_{h}(z)=f_{h}(x),
$$

where

$$
z=\frac{1}{2 x^{2}-1}
$$


Proof. Make the change of variable (2.10) in (2.7). Then $f_{h}(x)$ becomes

$$
F_{h}(z)=\sqrt{\frac{1-z^{2}}{z}}+\frac{1}{2} \int_{1}^{z} \frac{\mathrm{d} s}{s^{3 / 2} \sqrt{1-s^{2}}}
$$

in terms of the new variable $z=1 /\left(2 x^{2}-1\right)$. Since

$$
\frac{\mathrm{d}}{\mathrm{d} s} \sqrt{\frac{1-s^{2}}{s}}=\frac{-1-s^{2}}{2 s^{3 / 2} \sqrt{1-s^{2}}},
$$

integrating from 1 to $z$ reduces (2.11) to (2.8).

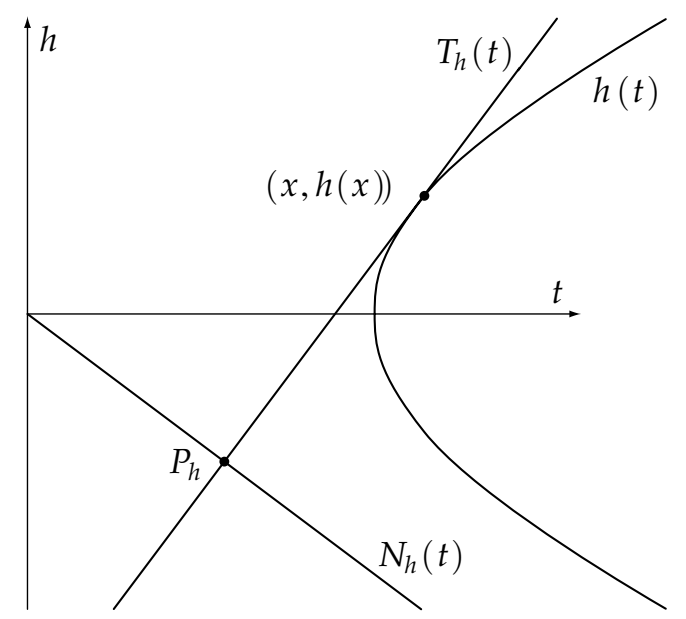

Fig. 1 The hyperbola

\section{The ellipse}

The equation of the ellipse can be written as

$$
e(t)=\sqrt{2\left(1-t^{2}\right)}, \quad|t| \leq 1
$$

In this case the tangent line at $t=r$ is

$$
T_{e}(t)=\sqrt{2\left(1-r^{2}\right)}-\sqrt{\frac{2 r^{2}}{1-r^{2}}}(t-r),
$$

and the line

$$
N_{e}(t)=\sqrt{\frac{1-r^{2}}{2 r^{2}}} t
$$


is the perpendicular to $T_{e}$ through the origin. These two lines intersect at the point

$$
P_{e}=\left(\frac{2 r}{1+r^{2}}, \frac{\sqrt{r\left(1-r^{2}\right)}}{1+r^{2}}\right)
$$

and the distance from $(r, e(r))$ to the common point $P_{e}$ is

$$
g_{e}(r)=r \sqrt{\frac{1-r^{2}}{1+r^{2}}} .
$$

We express the function $g_{e}$ in terms of the new variable $z=r^{2}$ as

$$
g_{e}(z)=\sqrt{\frac{z(1-z)}{1+z}}
$$

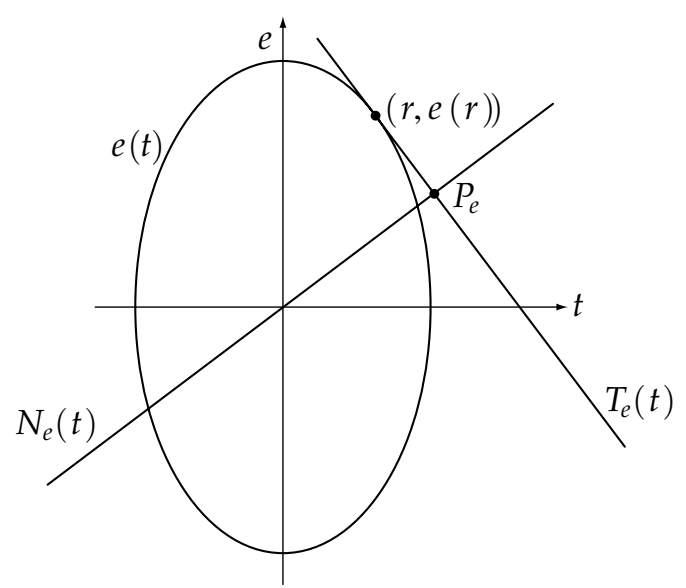

Fig. 2 The ellipse

\section{The connection}

We now evaluate the function $F_{h}(z)$ in $(2.8)$ at two points $y, z \in(0,1)$ related via the bilinear transformation $z=(1-y) /(1+y)$. We have

$$
F_{h}(z)+F_{h}(y)=\frac{1}{2} \int_{y}^{1} \sqrt{\frac{s}{1-s^{2}}} \mathrm{~d} s+\frac{1}{2} \int_{z}^{1} \sqrt{\frac{s}{1-s^{2}}} \mathrm{~d} s .
$$

The change of variable $\sigma=(1-s) /(1+s)$ in the second integral yields

$$
F_{h}(z)+F_{h}(y)=\frac{1}{2} \int_{y}^{1} \sqrt{\frac{s}{1-s^{2}}} \mathrm{~d} s+\frac{1}{2} \int_{0}^{y} \frac{\sqrt{1-\sigma}}{(1+\sigma)^{3 / 2} \sqrt{\sigma}} \mathrm{d} \sigma .
$$


Now recall the function $g_{e}(z)$ in (3.4) and its differential

Therefore

$$
\frac{\mathrm{d} g_{e}}{\mathrm{~d} z}=\frac{1}{2} \frac{\sqrt{1-z}}{\sqrt{z}(1+z)^{3 / 2}}-\frac{1}{2} \frac{\sqrt{z}}{\sqrt{1-z^{2}}} .
$$

$$
F_{h}(z)+F_{h}(y)=g_{e}(z)-g_{e}(1)+\frac{1}{2} \int_{0}^{1} \frac{\sqrt{t}}{\sqrt{1-t^{2}}} \mathrm{~d} t .
$$

Now observe that $g_{e}(1)=0$ and introduce the absolute constant

$$
L:=\frac{1}{2} \int_{0}^{1} \frac{\sqrt{t}}{\sqrt{1-t^{2}}} \mathrm{~d} t
$$

so that

$$
F_{h}(z)+F_{h}(y)=g_{e}(z)+L .
$$

Thus we have established the following integral relation.

Theorem 4.1 Let $y \in(0,1)$ and $z=(1-y) /(1+y)$. Then

$$
\frac{1}{2} \int_{y}^{1} \sqrt{\frac{s}{1-s^{2}}} \mathrm{~d} s+\frac{1}{2} \int_{z}^{1} \sqrt{\frac{s}{1-s^{2}}} \mathrm{~d} s=\sqrt{\frac{z(1-z)}{1+z}}+L
$$

with the absolute constant $L$ in (4.1).

Proof. Let

$$
\begin{aligned}
G_{h}(z) & =F_{h}(z)+F_{h}(y) \\
& =\frac{1}{2} \int_{(1-z) /(1+z)}^{1} \sqrt{\frac{s}{1-s^{2}}} \mathrm{~d} s+\frac{1}{2} \int_{z}^{1} \sqrt{\frac{s}{1-s^{2}}} \mathrm{~d} s,
\end{aligned}
$$

so that

$$
\frac{\mathrm{d} G_{h}(z)}{\mathrm{d} z}=\frac{1}{2} \frac{\sqrt{1-z}}{\sqrt{z}(1+z)^{3 / 2}}-\frac{1}{2} \frac{\sqrt{z}}{\sqrt{1-z^{2}}} .
$$

Integrating (4.4) gives

$$
G_{h}(z)=\sqrt{\frac{z(1-z)}{1+z}}+L .
$$

By letting $z=0$, the constant $L$ is easily evaluated as

$$
\begin{aligned}
L & :=\frac{1}{2} \int_{0}^{1} \frac{\sqrt{t}}{\sqrt{1-t^{2}}} \mathrm{~d} t \\
& =\frac{1}{2} \int_{0}^{\pi / 2} \sqrt{\sin \theta} \mathrm{d} \theta=\frac{\pi \sqrt{2 \pi}}{\Gamma^{2}(1 / 4)}
\end{aligned}
$$

using Wallis' formula. 
We now follow Landen to establish the value of $L$ in terms of elliptic arcs.

The equation (4.2) simplifies if we evaluate it at the fixed point $z^{*}=\sqrt{2}-1$ of the transformation $z=(1-y) /(1+y)$. In terms of the $x$ variable, the fixed point is

$$
x^{*}=\sqrt{1+\frac{1}{\sqrt{2}}}=\sqrt{2} \cos (\pi / 8) .
$$

Indeed

$$
F_{h}\left(z^{*}\right)=\frac{1}{2}(\sqrt{2}-1+L)
$$

Now introduce the complementary integral

$$
M:=\frac{1}{2} \int_{0}^{1} \frac{\mathrm{d} t}{\sqrt{t\left(1-t^{2}\right)}}
$$

and observe that

$$
L+M=L_{e}(1)=\frac{1}{2} \int_{0}^{1} \sqrt{\frac{1+t}{t(1-t)}} \mathrm{d} t
$$

where $L_{e}(1)$ is a quarter of the length of the ellipse.

Theorem 4.2 The integrals $L$ and $M$ satisfy

$$
\begin{aligned}
& L+M=L_{e}(1) \\
& L \times M=\frac{\pi}{4} .
\end{aligned}
$$

Therefore

$$
\begin{aligned}
L & =\frac{1}{2}\left(L_{e}(1)-\sqrt{L_{e}(1)^{2}-\pi}\right), \\
M & =\frac{1}{2}\left(L_{e}(1)+\sqrt{L_{e}(1)^{2}-\pi}\right) .
\end{aligned}
$$

Proof. Observe that for $q \in \mathbb{Q}$ we have

$$
\frac{\mathrm{d}\left(t^{q} \sqrt{1-t^{2}}\right)}{\mathrm{d} t}=\frac{q t^{q-1}-(q+1) t^{q+1}}{\sqrt{1-t^{2}}}
$$

and integrating from 0 to 1 we obtain

$$
\int_{0}^{1} \frac{t^{q-1}}{\sqrt{1-t^{2}}} \mathrm{~d} t=\frac{q+1}{q} \int_{0}^{1} \frac{t^{q+1}}{\sqrt{1-t^{2}}} \mathrm{~d} t .
$$

The proof now proceeds along the same line as Theorem 3.1 in [4].

We now write $\pi / 2=L_{c}(1)$ as a quarter of the length of the circle in analogy to $L_{e}(1)$. 
Theorem 4.3 The length of the hyperbolic segment is given by

$$
L_{h}\left(\frac{1}{\sqrt{2-\sqrt{2}}}\right)=\frac{\sqrt{2}+1}{2}-\frac{1}{4} \sqrt{\left(L_{e}(1)^{2}-4 L_{c}(1)\right)}-L_{e}(1) \text {. }
$$

\section{References}

[1] Cajori, F.: A history of the conceptions of limits and fluxions in Great Britain from Newton to Woodhouse. Open Court, Chicago 1919.

[2] Euler, L.: De miris proprietatibus curvae elasticae sub aequatione $y=\int x x / \sqrt{1-x^{4}} d x$ contentae, Comment 605 Enestroemianus Index. Acta academia scientiarum Petrop. 1782: II (1786), 34-61. Reprinted in Opera Omnia, ser. 1, 21, 91-118.

[3] Landen, J.: A disquisition concerning certain fluents, which are assignable by the arcs of the conic sections; wherein are investigated some new and useful theorems for computing such fluents. Philos. Trans. Roy. Soc. London 61 (1771), 298-309.

[4] Moll, V.H., Neill, P.A., Nowalsky, J.L., Solanilla, L.: A property of Euler's elastic curve. Elem. Math 55 (2000), 156-162.

[5] Nowalsky, J.L.: Properties of the Generalized Euler's Elastic Curve $y=\int x^{n} / \sqrt{1-x^{2 n}} d x$. Master Thesis, Tulane University, 1998.

Victor H. Moll

Department of Mathematics, Tulane University

New Orleans, Louisiana 70118, USA

e-mail: vhm@math.tulane.edu

Judith L. Nowalsky

Department of Mathematics, University of New Orleans

New Orleans, Louisiana 70148, USA

e-mail: jnowalski@math.uno.edu

Leonardo Solanilla

Departamento de Matemáticas,

Universidad del Tolima

Ibagué, Colombia

e-mail: solanila@bunde.tolinet.com.co

To access this journal online:

[4] http://www.birkhauser.ch 